$26 \mid 2009$

Varia

\title{
Le Néolithique
}

Cyril Marcigny

\section{OpenEdition}

Journals

Édition électronique

URL : http://journals.openedition.org/rao/943

DOI : $10.4000 /$ rao.943

ISBN : 978-2-7535-1609-0

ISSN : 1775-3732

Éditeur

Presses universitaires de Rennes

Édition imprimée

Date de publication : 31 décembre 2009

Pagination : 313-314

ISBN : 978-2-7535-1086-9

ISSN : 0767-709X

\section{Référence électronique}

Cyril Marcigny, «Le Néolithique», Revue archéologique de l'Ouest [En ligne], 26 | 2009, mis en ligne le 31 décembre 2011, consulté le 04 décembre 2020. URL : http://journals.openedition.org/rao/943 ; DOI : https://doi.org/10.4000/rao.943 
Après une étude de la céramique attestant de son attribution chronoculturelle au Néolithique final, la dernière partie, rédigée par Grégor Marchand et Sylvène Michel, replace les séries de l'Essart dans leur contexte chronoculturel global du premier et du deuxième Mésolithique. Ils réalisent là une synthèse approfondie qui complète les données déjà publiées par les deux auteurs.

La conclusion enfin reprend quelques aspects ethnographiques de la principale composante du site : les feux, en valorisant leur situation au bord du Clain.
D'un point de vue économique et social, les apports de la fouille et de la publication sont énormes et rappellent la complexité de ces ultimes chasseurs-cueilleurs. Grégor Marchand signe là un des ouvrages monographiques de référence sur la période pour le nord de la France avec, il ne faut pas l'oublier, une des plus grosses série d'armatures issue de fouilles.

Emmanuel GHESQUIÈre

(INRAP Basse-Normandie et UMR6566 - CreAAH)

TARrÊte, J. et Le Roux, C.-T. (coord.), 2008 - Le Néolithique, Paris, ministère de la Culture et de la Communication/ Picard, 424 p. (ISBN 978-2-7084-0801-2; $110 €$ ).

L’ouvrage, coordonné par Jacques Tarrête et CharlesTanguy Le Roux et qui s'ouvre sur une préface de Jean Guilaine, inaugure une nouvelle collection («Archéologie de la France »), coéditée par les éditions Picard et le Ministère de la Culture et de la Communication (Direction de l'Architecture et du Patrimoine) : Il s'agit d'un volumineux ouvrage grand format $(28 \times 24 \mathrm{~cm})$ de 415 pages (plus un index des sites archéologiques), qui fait le bilan des acquis récents de la recherche sur le Néolithique de la métropole et dans une moindre mesure de la Corse. Il réunit les articles d'une trentaine d'auteurs et d'une vingtaine de collaborateurs issus de l'ensemble du " paysage archéologique français » (CNRS surtout, suivi des agents de l'État, de l'université, des collectivités territoriales, puis de l'INRAP), qui explorent tour à tour les grands domaines d'étude de la Préhistoire récente : le rapport de l'homme à son environnement, l'habitat et l'organisation sociale, les vestiges mobiliers (fabrication, matériaux), les pratiques funéraires, les relations sociales et les manifestations artistiques. Les chercheurs mis à contribution sont parmi les meilleurs dans leurs spécialités et offrent des synthèses très richement illustrées (il s'agit ici d'un « beau livre »), ponctuées par des encarts consacrés aux méthodes mises à contribution pour la recherche en Préhistoire : datations radiocarbone, analyse chimique des matériaux, tracéologie, expérimentation, analyse $\mathrm{ADN}$, etc.

Les chapitres sont précédés d'une préface de Jean Guilaine. Véritable introduction à l'ouvrage, elle replace le Néolithique français dans le vaste mouvement qui couvre le Proche Orient et une bonne partie de l'Europe. L'auteur y évoque, dans de courts bilans historiographiques, les principales avancées dans chacune des thématiques qui forment les chapitres du livre. C'est aussi pour ce chercheur l'occasion de proposer de nouvelles pistes d'études et de partager avec le lecteur ses souhaits en matière d'évolution de la recherche.
La préface est suivie de six cartes permettant de saisir l'emprise des groupes néolithiques entre les $\mathrm{VI}^{e}$ et $\mathrm{III}^{\mathrm{e}}$ millénaires. Cet exercice cartographique, des plus périlleux lorsque l'on sait à quel point les néolithiciens peuvent se quereller à propos de la répartition des groupes culturels au cours du temps, n'échappera pas à la critique. Plutôt que de fustiger ce travail, il faut plutôt examiner ces cartes comme un état de la recherche et un bel effort de synthèse formant une bien utile introduction pour qui veut poursuivre plus avant la lecture de l'ouvrage. On regrettera toutefois ici l'absence d'un tableau chronologique reprenant ces données de manière synthétique. En effet, le seul cadre chronologique proposé avant la lecture du corps de l'ouvrage va du Tardiglaciaire à nos jours et a pour objet les évolutions environnementales.

Les 26 chapitres qui suivent sont regroupés en cinq grandes parties. Ils traitent de l'ensemble des données disponibles sur le Néolithique en laissant souvent une large place à l'interdisciplinarité, fait encore trop rarement mis en avant dans les synthèses parues ces dernières années qui font généralement la part belle à la seule culture matérielle. Certains pourront, bien entendu, pointer du doigt les lacunes ou les absences dans certains chapitres (en particulier la relative discrétion des données issues de l'archéologie préventive). D'autres pourront incriminer le choix des auteurs, certains étant absents du " casting " alors qu' ils font figure de références internationales; il reste que ce travail est monumental. C'est l'aboutissement d'un projet lancé il y a plus de 10 ans; malgré cette longue gésine qui pourrait laisser présager un ouvrage obsolète, il est important de souligner que la plupart des chapitres ont été réactualisés par leurs auteurs ces dernières années (les premières livraisons datent de 2000, beaucoup ont été réécrites en 2005 et 2006), permettant donc aux lecteurs de profiter des dernières avancées scientifiques dans certains domaines. 
Au final, certains articles sont très pointus, d'autres plus généraux, les illustrations sont abondantes (et en couleurs). L'ensemble est agréable à lire et bien documenté. Les articles renvoient bien souvent à une bibliographie dense permettant de poursuivre sa lecture et d'approfondir ses connaissances, thématique par thématique. Seul ombre au tableau, il est difficile de savoir quelle est la "cible de vente " de cette nouvelle collection. Il ne s'agit ni d'une véritable synthèse à destination de la communauté scientifique (bien que nombre de professionnels y trouveront matière à réflexion), ni d'un ouvrage accessible facilement au grand public (beaucoup de termes ne sont pas explicités par manque de place). En fait ce panorama du Néolithique-formerait un très bon manuel à destination des étudiants, mais le prix de 110 euros risque d'en rebuter plus d'un, à juste titre. Il ne reste plus qu'à espérer que toutes les bibliothèques universitaires (et les autres) fassent l'achat de ce livre de référence.

Cyril MarcignY

(INRAP Basse-Normandie et UMR6566 - CReAAH)

Kern, A., Kowarik, K., Rausch, A. W. et Reschreiter H., 2008 - Salz-Reich. 7000 Jahre Hallstatt, Vienne, éditions du Musée d'Histoire naturelle, 240 p. (ISBN 978-3-902421-26-5; $25 €$ ).

Cet ouvrage collectif en langue allemande est édité par le Musée d'Histoire naturelle de Vienne où se trouve actuellement conservée l'impressionnante collection archéologique issue du site de Hallstatt en Autriche.

Ce site éponyme, qui a donné son nom à la période du $1^{\text {er }}$ Âge du Fer mais qui aurait aussi bien pu lui valoir le titre de "Âge du Sel », est l'un des complexes archéologique majeurs de la protohistoire européenne. Depuis 7000 ans, le destin des hommes de cette région est définitivement lié à cette ressource de l'or blanc et à l'exploitation du sel gemme qui sommeille au cœur du massif du Salzkammergut dans les Préalpes autrichiennes. La petite ville de Hallstatt et la région Dachstein/Salzkammergut ont d'ailleurs été inscrites au titre du Patrimoine mondial culturel et naturel de l'Unesco en 1997.

Enchaînant une quinzaine de notices d'inégales longueurs et de portée scientifique variable, l'organisation générale du propos échappe au premier abord et évoque davantage un catalogue d'exposition qu'un ouvrage de synthèse. L'ouvrage dresse le tableau des formes d'exploitation des mines de sel à travers la dynamique de formation des gisements et les procédés techniques d'extraction successivement mis en œuvre, depuis l'Âge du Bronze jusqu'à l'époque moderne. L'organisation du travail au sein des divers puits et galeries de mines et leurs aménagements internes en bois sont notamment analysés en détail pour la fin de la Préhistoire. La vie des mineurs protohistoriques, la culture matérielle et les outillages sont bien entendus illustrés à partir des vestiges archéologiques remarquablement conservés dans les galeries de mines : outillage en bois, métal, pierre (pics de mineurs, masses, flambeaux...), vêtements et tenues de travail en cuir ou en tissus (hottes de mineurs, gants, casque, chaussures, etc.), vaisselle de bois, céramiques... L'un des chapitres revient également sur la remarquable collection d'objets archéologiques importés de diverses régions d'Europe et issue de la nécropole de l'Âge du Fer.

L’une des qualités de l'ouvrage est de décliner les diverses thématiques en synthétisant les données récentes et celles des fouilles anciennes, dont les travaux pionniers de Georg Ramsauer qui, à partir de 1846 et pendant 17 ans, explora 980 tombes d'où il exhuma plus de 19000 objets (dont la plupart des objets de prestige circulant à l'époque en Europe).

L'une des caractéristiques du site étant l'incroyable collection d'objets et macro-restes organiques, la présentation des données les plus récentes fait intervenir divers champs disciplinaires et approches complémentaires, de l'archéobotanique à la dendrologie et l'archéologie expérimentale en passant par la parasitologie, les analyses de textiles et l'archéozoologie, qui offrent des résultats parfois spectaculaires et sont autant d'occasion pour exposer, de manière très pédagogique, tout un panel de méthodes de travail.

Une autre qualité de l'ouvrage est l'abondance et la qualité des illustrations, avec plus de 600 documents en couleurs au total, dont une large partie relève d'une documentation inédite.

Marie-Yvane DAIRE 